\title{
Quasi-Degenerate Neutrino Masses with Normal and Inverted Hierarchy
}

\author{
Ngouniba Ki Francis $^{{ }^{*} \text {, }}$ Ngangkham Nimai Singh ${ }^{2}$ \\ ${ }^{1}$ Department of Physics, Tezpur University, Tezpur, India \\ ${ }^{2}$ Department of Physics, Gauhati University, Guwahati, India \\ E-mail: ${ }^{*}$ ngkf2010@gmail.com \\ Received June 10, 2011; revised July 28, 2011; accepted August 16, 2011
}

\begin{abstract}
The effects of CP-phases on the three absolute quasi-degenerate Majorana neutrino (QDN) masses are studied with neutrino mass matrices obeying $\mu-\tau$ symmetry for normal as well as inverted hierarchical mass patterns. We have made further investigations on 1) the prediction of solar mixing angle which lies below tri-bimaximal mixing value in consistent with neutrino oscillation observational data, 2) the prediction on absolute neutrino mass parameter $\left(m_{e e}\right)$ in $0 v \beta \beta$ decay, and 3) cosmological bound on the sum of the three absolute neutrino masses $\sum_{i}^{3} m_{i}$. The numerical analysis is carried out through the parameterization of neutrino mass matrices using only two unknown parameters $(\varepsilon, \eta)$ within $\mu-\tau$ symmetry. The results show the validity of QDN mass models in both normal and inverted hierarchical patterns. These models are far from discrimination and hence not yet ruled out. The results presented in this article are new and have subtle effects in the discrimination of neutrino mass models.
\end{abstract}

Keywords: QDN Models, Absolute Neutrino Masses, CP-Phases

\section{Introduction}

The presently available neutrino oscillation observational data [1-5] on two neutrino mass-squared differences $\left(\Delta m_{21}^{2},\left|\Delta m_{23}^{2}\right|\right)$, is found to be insufficient to predict the three absolute neutrino masses in case of quasi-degenerate neutrino (QDN) mass models [6-14], as three independent equations are required for solving three unknown values of absolute neutrino masses. The values of the absolute neutrino mass scale ranging from $0.1 \mathrm{eV}$ to $0.4 \mathrm{eV}$ have been taken as input parameters in most of the theoretical calculations [6-15] in the literature. However, since the present tightest cosmological upper bound of the sum of three absolute neutrino masses, has gone down to the lowest value, $\sum m_{i} \leq 0.28 \mathrm{eV}[16,17]$, a larger value of neutrino mass $m_{i} \geq 0.1 \mathrm{eV}$ in QDN models, has to be ruled out. Further the upper bound value of neutrino mass parameter $m_{e e} \leq 0.27 \mathrm{eV}$ appeared in the neutrinoless double beta decay $(0 v \beta \beta)$ experiments [18], also disfavours larger values of neutrino mass eigenvalues with same CP-parity. Investigations on QDN models for both normal hierarchical (NH) and inverted hierar- chical (IH) patterns of the three absolute neutrino masses, require detailed numerical analysis to check whether such QDN models can really accommodate lower values of absolute neutrino masses $m_{i} \leq 0.09 \mathrm{eV}$ which is consistent with the above cosmological bound. In addition, the solar mixing angle lying below the tri-bimaximal mixing (TBM) [19-21], which is consistent with present observational data [1-5] and the effects of CP-phases on three absolute neutrino masses, are also important ingredients for further analysis of QDN models. We address all these issues in the present work and show the validity of the quasi-degenerate models for lower values of neutrino masses $m_{i} \leq 0.09 \mathrm{eV}$. This constitutes the main part of our work which differs from others on the mass scale rather than on the resulting lower solar mixing angles. We first introduce a general classification of QDN models based on their CP-parity patterns in the three absolute neutrino masses, and then parameterize the mass matrices using only two unknown parameters $(\varepsilon, \eta)$ for achieving a practical numerical solution within $\mu-\tau$ symmetry. Finally we present a detailed numerical analysis and results. 


\section{Parameterizations of Neutrino Mass Matrix}

A general $\mu-\tau$ symmetric neutrino mass matrix [22-24] with its four unknown independent matrix elements, requires at least four independent equations for a realistic numerical solution,

$$
m_{L L}=\left(\begin{array}{lll}
m_{11} & m_{12} & m_{12} \\
m_{12} & m_{22} & m_{23} \\
m_{12} & m_{23} & m_{22}
\end{array}\right) .
$$

The three mass eigenvalues $m_{i}$ and solar mixing angles $\theta_{12}$, are given by

$$
\begin{aligned}
& m_{1}=m_{11}-\sqrt{2} \tan \theta_{12} m_{12}, \\
& m_{2}=m_{11}+\sqrt{2} \cot \theta_{12} m_{12}, \\
& m_{3}=m_{22}-m_{23} . \\
& \tan 2 \theta_{12}=\frac{2 \sqrt{2} m_{12}}{m_{11}-m_{22}-m_{23}} .
\end{aligned}
$$

The observed mass-squared differences are then calculated as

$$
\begin{aligned}
& \Delta m_{12}^{2}=m_{2}^{2}-m_{1}^{2}>0 \\
& \Delta m_{32}^{2}=\left|m_{3}^{2}-m_{2}^{2}\right| .
\end{aligned}
$$

In the basis where charged lepton mass matrix is diagonal, we have the leptonic mixing matrix $U_{P M N S}=U$ of the form $[23,24]$,

$$
U_{P M N S}=\left(\begin{array}{ccc}
\cos \theta_{12} & -\sin \theta_{12} & 0 \\
\frac{\sin \theta_{12}}{\sqrt{2}} & \frac{\cos \theta_{12}}{\sqrt{2}} & -\frac{1}{\sqrt{2}} \\
\frac{\sin \theta_{12}}{\sqrt{2}} & \frac{\cos \theta_{12}}{\sqrt{2}} & \frac{1}{\sqrt{2}}
\end{array}\right)
$$

The neutrino mass parameters $m_{e e}$ appeared in the neutrinoless double beta decay $(0 v \beta \beta)$ and the sum of the three absolute neutrino masses in WMAP cosmological bound $\sum_{i}^{3} m_{i}$, are given respectively by,

$$
\begin{aligned}
& m_{e e}=\left|m_{1} U_{e 1}^{2}+m_{2} U_{e 2}^{2}+m_{3} U_{e 3}^{2}\right|, \\
& m_{\text {cosmos }}=m_{1}+m_{2}+m_{3} .
\end{aligned}
$$

A general classification for the three types of quasidegenerate neutrino mass models [22] with respect to the Majorana CP-phases in their three mass eigenvalues, is adopted here. Diagonalisation of the left-handed Majorana neutrino mass matrix $m_{L L}$ in Equation (1) is given by $m_{L L}=U D U^{T}$, where $U$ is the diagonalising matrix in
Equation (4) and $D=\operatorname{Diag}\left(m_{1}, m_{2} e^{i \phi_{1}}, m_{3} e^{i \phi_{2}}\right)$ is the diagonal matrix with two unknown Majorana phases $\left(\phi_{1}, \phi_{2}\right)$. In the basis where charged lepton mass matrix is diagonal, the leptonic mixing matrix is given by $U=U_{P M N S}[23,24]$. We then adopt the following classification according to their CP-parity patterns in the mass eigenvalues $m_{i}$, namely, Type IA: $(+-+)$ for $D=\operatorname{Diag}\left(m_{1},-m_{2}, m_{3}\right)$; Type IB: $(+++)$ for $D=\operatorname{Diag}\left(m_{1}, m_{2}, m_{3}\right)$ and Type IC: $(++-)$ for $D=\operatorname{Diag}\left(m_{1}, m_{2},-m_{3}\right)$ respectively. We now introduce the following parameterization of $\mu-\tau$ symmetric neutrino mass matrices $m_{L L}$ which satisfy the above classifications [22].

\section{Numerical Analysis and Results}

We first estimate the numerical values of the three absolute neutrino masses. As discussed before, we need to introduce the neutrino mass scale $m_{3}$ as input parameter in addition to the observational data [1-5] on solar and atmospheric neutrino mass-squared differences $\left(\Delta m_{21}^{2}\right.$ and $\left|\Delta m_{32}^{2}\right|$ ). For numerical analysis we use the best-fit values of the global neutrino oscillation observational data $[1-5]$

$$
\Delta m_{12}^{2}=\left(m_{2}^{2}-m_{1}^{2}\right)=7.60 \times 10^{-5} \mathrm{eV}^{2},
$$

and

$$
\left|\Delta m_{32}^{2}\right|=\left|m_{3}^{2}-m_{2}^{2}\right|=2.40 \times 10^{-3} \mathrm{eV}^{2},
$$

and also define the following parameters $\alpha=\frac{\left|\Delta m_{23}^{2}\right|}{m_{3}^{2}}$ and $\beta=\frac{\Delta m_{21}^{2}}{\left|\Delta m_{23}^{2}\right|}$, where $m_{3}$ is the input quantity. For quasidegenerate model with normal hierarchy (NH-QD), the other two mass eigenvalues are estimated from,

$$
\begin{aligned}
& m_{2}=m_{3} \sqrt{1-\alpha} ; \\
& m_{1}=m_{3} \sqrt{1-\alpha(1+\beta)}
\end{aligned}
$$

and for quasi-degenerate model with inverted hierarchy (IH-QD) the mass eigenvalues are extracted from,

$$
\begin{aligned}
& m_{2}=m_{3} \sqrt{1-\alpha} ; \\
& m_{1}=m_{3} \sqrt{1+\alpha(1-\beta)} .
\end{aligned}
$$

For suitable input value of $m_{3}$, one can estimate the values of $m_{1}$ and $m_{2}$ for both NH-QD and IH-QD models, using the observational values of $\Delta m_{21}^{2}$ and $\left|\Delta m_{23}^{2}\right|$. Table 1 gives the calculated numerical values for both models.

In the next step we parameterize the mass matrix Equation (1) into three types: Type IA with 
$D=\operatorname{Diag}\left(m_{1},-m_{2}, m_{3}\right)$ : The mass matrix of this type [22, $25,26]$ can be parameterized using two parameters $(\varepsilon, \eta)$ :

$$
m_{L L}=\left(\begin{array}{ccc}
\varepsilon-2 \eta & -c \varepsilon & -c \varepsilon \\
-c \varepsilon & \frac{1}{2}-d \eta & -\frac{1}{2}-\eta \\
-c \varepsilon & -\frac{1}{2}-\eta & \frac{1}{2}-d \eta
\end{array}\right) m_{3} .
$$

This predicts the solar mixing angle,

$$
\tan 2 \theta_{12}=-\frac{2 c \sqrt{2}}{1+(d-1) \eta / \varepsilon} .
$$

When we choose the values $c=d=1.0$, we get the tribimaximal mixings (TBM), $\tan 2 \theta_{12}=-2 \sqrt{2}$ (which is $\left.\tan ^{2} \theta_{12}=0.50\right)$ and the values of $\varepsilon$ and $\eta$ are calculated for both NH-QD and IH-QD models, by using the values of Table 1 in these two eigenvalue expressions: $m_{1}=$ $(2 \varepsilon-2 \eta) m_{3}$ and $m_{2}=(\varepsilon-2 \eta) m_{3}$. The results are given in Table 2 for $\tan ^{2} \theta_{12}=0.50$. The solar angle can be further lowered by taking the values $c<1$ and $d>1$ while using the earlier values of $\varepsilon$ and $\eta$ extracted using
TBM. For $\tan ^{2} \theta_{12}=0.45$ case, the results are given in Table 3 Type IB with $D=\left(m_{1}, m_{2}, m_{3}\right)$ : This type [22,25, $26]$ of quasidegenerate mass pattern is given by the mass matrix,

$$
m_{L L}=\left(\begin{array}{ccc}
1-\varepsilon-2 \eta & c \varepsilon & c \varepsilon \\
c \varepsilon & 1-d \eta & -\eta \\
c \varepsilon & -\eta & 1-d \eta
\end{array}\right) m_{3}
$$

This predicts the solar mixing angle,

$$
\tan 2 \theta_{12}=\frac{2 c \sqrt{2}}{1+(1-d) \eta / \varepsilon},
$$

which gives the TBM solar mixing angle with the input values $c=1.0$ and $d=1.0$. Like in Type-IA, here $\varepsilon$ and $\eta$ values are computed for NH-QD and IH-QD, by using Table 1 in the corresponding two eigenvalue expressions: $m_{1}=(1-2 \varepsilon-2 \eta) m_{3}$ and $m_{2}=(1+\varepsilon-2 \eta) m_{3}$. Type IC with $D=\left(m_{1}, m_{2},-m_{3}\right)$ : It is not necessary to treat this model [22] separately as it is similar to Type IB except

Table 1. The absolute neutrino masses in eV estimated from oscillation data [1] (Using calculated value of $\beta=0.03166$ ).

\begin{tabular}{cccccc}
\hline Input & Calculated & \multicolumn{2}{c}{ NH-QD } & \multicolumn{2}{c}{ IH-QD } \\
\hline $\boldsymbol{m}_{\mathbf{3}}$ & $\boldsymbol{\alpha}$ & $\boldsymbol{m}_{\mathbf{1}}$ & $\boldsymbol{m}_{\mathbf{2}}$ & $\boldsymbol{m}_{\mathbf{1}}$ & $\boldsymbol{m}_{\mathbf{2}}$ \\
\hline 0.400 & 0.015 & 0.3968929 & 0.39699 & 0.4028945 & 0.4029888 \\
0.10 & 0.24 & 0.086741 & 0.08718 & 0.111035 & 0.1113552 \\
0.08 & 0.375 & 0.0626418 & 0.06326 & 0.0934023 & 0.0938083 \\
\hline
\end{tabular}

Table 2. Prediction for $\tan ^{2} \theta_{12}=0.50$.

\begin{tabular}{ccccc}
\hline \multirow{2}{*}{ Parameters } & \multicolumn{2}{c}{ NH-QD } & \multicolumn{2}{c}{ IH-QD } \\
\cline { 2 - 5 } & Type-IA & Type-IB & Type-IA & Type-IB \\
\hline$c$ & 1.0 & 1.0 & 1.0 & 1.0 \\
$d$ & 1.0 & 1.0 & 0.10 & 0.10 \\
$m_{3}(\mathrm{eV})$ & 0.10 & 0.10 & 0.08 & 0.08 \\
$\varepsilon$ & 0.57972 & 0.0015 & 0.78004 & 0.00169 \\
$\eta$ & 0.14602 & 0.0649 & 0.19628 & -0.0855 \\
\hline$m_{1}(\mathrm{eV})$ & 0.08674 & 0.08672 & 0.09340 & 0.09340 \\
$m_{2}(\mathrm{eV})$ & -0.0872 & 0.08717 & -0.0938 & 0.09380 \\
$m_{3}(\mathrm{eV})$ & 0.10 & 0.10 & 0.08 & 0.08 \\
$\Sigma\left|m_{i}\right| \mathrm{eV}$ & 0.273 & 0.273 & 0.267 & 0.267 \\
\hline$\Delta m_{21}^{2} \mathrm{eV}$ & $7.6 \times 10^{-5}$ & $7.6 \times 10^{-5}$ & $7.6 \times 10^{-5}$ & $7.6 \times 10^{-5}$ \\
$\left|\Delta m_{23}^{2}\right| \mathrm{eV}$ & $2.4 \times 10^{-3}$ & $2.4 \times 10^{-3}$ & $2.4 \times 10^{-3}$ & $2.4 \times 10^{-3}$ \\
$\tan ^{2} \theta_{12}$ & 0.50 & 0.50 & 0.50 & 0.50 \\
$\left|m_{e e}\right| \mathrm{eV}$ & 0.0869 & 0.0869 & 0.0869 & 0.0869 \\
\hline
\end{tabular}


Table 3. Prediction for $\tan ^{2} \theta_{12}=0.45$

\begin{tabular}{ccccc}
\hline \multirow{2}{*}{ Parameters } & \multicolumn{2}{c}{ NH-QD } & IH-QD \\
\cline { 2 - 4 }$d$ & Type-IA & Type-IA & Type-IA & Type-IB \\
\hline$m_{3}(\mathrm{eV})$ & 0.86 & 0.945 & 0.868 & 0.96 \\
$\varepsilon$ & 1.025 & 0.998 & 1.0 & 0.08 \\
$\eta$ & 0.10 & 0.10 & 0.08 & 0.00169 \\
$m_{1}(\mathrm{eV})$ & 0.6616 & 0.00145 & 0.88762 & -0.0855 \\
$m_{2}(\mathrm{eV})$ & 0.1655 & 0.06483 & 0.22317 & 0.09341 \\
$m_{3}(\mathrm{eV})$ & 0.08754 & 0.08676 & 0.09392 & 0.09381 \\
$\Sigma\left|m_{i}\right| \mathrm{eV}$ & -0.0879 & 0.08717 & -0.0943 & 0.08001 \\
\hline$m_{21}^{2} \mathrm{eV}$ & 0.0996 & 0.10002 & 0.08 & 0.267 \\
$\left|\Delta m_{23}^{2}\right| \mathrm{eV}$ & 0.275 & 0.274 & 0.268 & $7.4 \times 10^{-5}$
\end{tabular}

for the interchange of two matrix elements $\{22\}$ and $\{23\}$ in the mass matrix in Equation (10), and this effectively imparts an additional odd CP-parity on the third mass eigenvalue $m_{3}$ in Type IC. Such change does not alter the predictions of Type IB. A self explanatory results for two models of Types-IA and IB, for $\tan ^{2} \theta_{12}=0.50$ and $\tan ^{2} \theta_{12}=0.45$, are presented in Tables 2 and 3 respectively.

\section{Conclusions}

We have studied the effects of the Majorana phases on the prediction of absolute neutrino masses in three different types of QDN models having both normal and inverted hierarchical patterns within $\mu-\tau$ symmetry. These predictions are consistent with observational data on the mass-squared difference derived from various neutrino oscillation experiments, and with the upper bound on absolute neutrino mass parameter in $0 v \beta \beta$ decay as well as upper bound of $\sum m_{i} \leq 0.28 \mathrm{eV}$ obtained from cosmological observations. It has been found that the QDN models with $m_{i} \leq 0.09 \mathrm{eV}$, are still far from discrimination and hence not yet ruled out. The prediction on solar mixing angle is also found to be lower than TBM value viz, $\tan ^{2} \theta_{12}=0.45$ which coincides with the best-fit in the neutrino oscillation data [1]. The result shows the validity of NH-QD and IH-QD models. The results presented in this article are new and have important implications in the discrimination of neutrino mass models.

\section{Acknowledgements}

Ng. K. F thanks the University Grants Commission, Government of India for sanctioning the project entitled "Neutrino masses and mixing angles in neutrino oscillations" vide Grant. No 32-64/2006 (SR). This work was carried out through this project.

\section{References}

[1] T. Schwetz, M. Tortola and J. W. F. Valle, "ThreeFlavour Neutrino Oscillation Update," New Journal of Physics, Vol. 10, 2008, p. 113011. doi:10.1088/1367-2630/10/11/113011

[2] T. Schwetz, M. Tortola and J. W. F. Valle, "Global Neutrino Data and Recent Reactor Fluxes: Status of Three Flavour Oscillation Parameters," New Journal of Physics, Vol. 13, 2011, p. 063004. doi:10.1088/1367-2630/13/6/063004

[3] M. C. Gonzales-Garcia, M. Maltoni and J. W. F. Valle, "Phenomenology with Massive Neutrinos," Physics Reports, Vol. 460, No. 1-3, 2008, pp. 1-129. doi:10.1016/j.physrep.2007.12.004

[4] M. C. Gonzales-Garcia, M. Maltoni and J. W. F. Valle, "Understanding and Probing Neutrino Oscillations," Invited Talk in Neutrino-2010, Athens, 14 June 2010;

[5] A. Bandyopadhyay, et al., "Physics at a Future Neutrino Factory and Super-Beam Facility," Reports on Progress in Physics, Vol. 72, 2009, p. 106201. doi:10.1088/0034-4885/72/10/106201

[6] E. M. Lipmanov, "Quasi-Degenerate Neutrino Masses in 
Terms of Mass Squared Differences," Physics Letters B, Vol. 567, 2003, pp. 268-272.

doi:10.1016/j.physlettb.2003.06.052

[7] A. S. Joshipura, "Neutrino Spectrum from Theory and Experiments," Pramana, Vol. 54, No. 1, 1994, pp. 119132. doi:10.1007/s12043-000-0011-7

[8] S. T. Petcov and A. Yu. Smirnov, "Neutrinoless Double Beta Decay and the Solar Neutrino Problem," Physics Letters B, Vol. 322, 1994, pp. 109-118. doi:10.1016/0370-2693(94)90498-7

[9] A. S. Joshipura and K. M. Patel, "Quasi-degenerate Neutrinos in SO (10)," Physical Review D, Vol. 82, 2010, p. 031701. doi:10.1103/PhysRevD.82.031701

[10] A. S. Joshipura, K. M. Patel and S. K. Vempti, "Type I Seesaw Mechanism for Quasi-Degenerate Neutrinos," Physics Letters B, Vol. 690, No. 3, 2010, pp. 289-295.

[11] S. Antusch and S. F. King, "From Hierarchical to Partially Degenerate Neutrinos via Type II Upgrade of Type I See-Saw Models," Nuclear Physics B, Vol. 705, 2005, pp. 239-268. doi:10.1016/j.nuclphysb.2004.10.049

[12] P. Binetruy, S. Lavignac, S. T. Petcov and P. Ramond, "Quasi-Degenerate Neutrinos from an Abelian Family Symmetry," Nuclear Physics B, Vol. 496, 1997, pp. 3-23.

[13] G. C. Branco, M. N. Rebelo and J. I. Silva-Marcos, "Degenerate and Quasi-Degenerate Majorana Neutrinos," Physical Review Letters, Vol. 82, No. 4, 1999, pp. 683686. doi:10.1103/PhysRevLett.82.683

[14] G. C. Branco, M. N. Rebelo and J. I. Silva-Marcos, "Quasi-Degenerate neutrino Masses with Universal strength Yukawa Coupling," Physics Letters B, Vol. 428, No. 1, 1998, pp. 136-142.

[15] I. M. Varzielas, G. G. Ross and M. Serna, "Quasidegenerate Neutrinos and Tri-Bimaximal Mixing," Physical Review D, Vol. 80, 2009, p. 073002. doi:10.1103/PhysRevD.80.073002

[16] S. A. Thomas, F. B. Abdalla and O. Lahav, "Upper Bound on $0.28 \mathrm{eV}$ on Neutrino Masses from the Largest Photometric Redshift Survey," Physical Review Letters, Vol. 105, 2010, p. 031301. doi:10.1103/PhysRevLett.105.031.301
[17] J. Lesgourgues, "Galaxies Weigh in on Neutrinos," Physics, Vol. 3, 2010, pp. 57-59. doi:10.1103/Physics.3.57

[18] S. Pascoli and S. T. Petcov, "Majorana Neutrinos, Neutrino Mass Spectrum and the $<\mathrm{M}>\sim 0.001 \mathrm{eV}$ Frontier in Neutrinoless Double Beta Decay," Physical Review D, Vol. 77, 2008, p. 113003. doi:10.1103/PhysRevD.77.113003

[19] P. F. Harrison and W. G. Scott, "Mu-Tau Reflection Symmetry in Lepton Mixing and Neutrino Oscillations," Physics Letters B, Vol. 547, No. 3, 2002, pp. 219-228. doi:10.1016/S0370-2693(02)02772-7

[20] P. F. Harrison, D. H. Perkins and W. G. Scott, "TriBimaximal Mixing and the Neutrino Oscillation Data," Physics Letters B, Vol. 530, 2002, pp. 167-178. doi:10.1016/S0370-2693(02)01336-9

[21] P. F. Harrison and W. G. Scott, "Permutation Symmetry, Tri-Bimaximal Neutrino Mixing and $\mathrm{S}_{3}$," Physics Letters $B$, Vol. 557, 2003, pp. 76-90. doi:10.1016/S0370-2693(03)00183-7

[22] G. Altarelli and F. Feruglio, "Neutrino Masses and Mixings: A Theoretical Perspective," Physical Report, Vol. 320, 1999, pp. 295-325. doi:10.1016/S0370-1573(99)00067-8

[23] Z. Maki, M. Nakagawa and S. Sakata, "Remarks on the Unified Model of Elementary Particles," Progress of Theoretical Physics, Vol. 28, No. 5, 1962, p. 870-880 doi:10.1143/PTP.28.870

[24] B. Pontecorvo, "Neutrino Experiments and the Problem of Conservation of Lepton Charge," Soviet PhysicsJETP, Vol. 26, 1968, pp. 984-988.

[25] N. Nimai-Singh, M. Rajkhowa and A. Borah, "Lowering Solar Mixing Angle in Inverted Hierarchy without Charged Lepton Corrections," Journal of Physics G: Nuclear and Particle Physics, Vol. 34, No. 2, 2007, pp. 345-351. doi:10.1088/0988-3899/34/2/013

[26] N. Nimai-Singh, M. Rajkhowa and A. Borah, "Deviation From Tri-Bimaximal Mixing Through Flavour Twisters in Inverted and Normal Hierarchical Neutrino Mass Models," Pramana, Vol. 69, No. 4, 2007, pp. 533-549. doi:10.1007/s12043-007-0154-X 